\title{
Consenso para el diagnóstico clínico y microbiológico y la prevención de la infección por Bordetella pertussis
}

\author{
Grupo de expertos en vacunación contra tos ferina*
}

\begin{abstract}
Grupo de expertos en vacunación contra tos ferina. Consenso para el diagnóstico clínico y microbiológico y la prevención de la infección por Bordetella pertussis. Salud Publica Mex 201 I;53:57-65.
\end{abstract}

\begin{abstract}
Resumen
La tos ferina sigue siendo responsable de una carga de enfermedad importante en el mundo. Aunque la implementación del uso de la vacuna contra esta enfermedad ha disminuido en gran medida el número de casos en la población pediátrica, se ha observado que la inmunidad inducida por la vacuna y por la infeccion natural disminuye con el tiempo lo que hace nuevamente susceptibles a adolescentes y adultos jóvenes que pueden transmitir la enfermedad a lactantes no inmunizados o con esquema de vacunación incompleto. Este documento, resultado de la reunión de un grupo internacional de expertos en la Ciudad de México, ha analizado la información médica reciente para establecer el estado actual de la epidemiología, diagnóstico, vigilancia y, especialmente, el valor de la dosis de refuerzo con dTpa en adolescentes y adultos como estrategia de prevención de tos ferina en México.
\end{abstract}

Palabras clave: tos ferina; Bordetella pertussis; vacunas contra difteria, tétanos $y$ tos ferina acelular
Expert group on vaccinatons for pertussis.

Consensus on the clinical and microbiologic diagnosis

of Bordetella pertussis, and infection prevention.

Salud Publica Mex 20I I;53:57-65.

\begin{abstract}
Pertussis continues to be responsible for a significant disease burden worldwide. Although immunization practices have reduced the occurrence of the disease among children, waning vaccine- and infection-induced immunity still allows the disease to affect adolescents and adults who, in turn, can transmit the disease to non-immunized or partially immunized infants. This document is the result of a meeting in Mexico City of international experts who analyzed recent medical information in order to establish the current status of the epidemiology, diagnosis and surveillance of pertussis and, especially, the value of the $\mathrm{dT}$ pa booster dose in adolescents and adults as a pertussis prevention strategy in Mexico.
\end{abstract}

Key words: pertussis; Bordetella pertussis; diphtheria-tetanusacellular pertussis vaccines

\footnotetext{
* Integrantes (en orden alfabético): Sandra Beltrán Silva, Hospital General de Tuxtla Gutiérrez, Chiapas, México; Yolanda Cervantes Apolinar, Dirección Médica Vacunas, GlaxoSmithKline México;James D. Cherry, David Geffen School of Medicine at UCLA and Mattel Children's Hospital,UCLA, California, Estados Unidos; Carlos Conde González, Centro de Investigación sobre Enfermedades Infecciosas, Instituto Nacional de Salud Pública, México; Angela Gentile, Jefa de Epidemiología del Hospital de Niños Ricardo Gutiérrez, Buenos Aires, Argentina; César Misael Gómez Altamirano, Centro de Atención para la Salud de la Infancia y la Adolescencia (CENSIA), Secretaría de Salud, México; Marte Hernández Porras, Instituto Nacional de Pediatría, México; Gloria Huerta García, Hospital de Pediatría, Centro Médico Nacional Siglo XXI, IMSS, México; Mercedes Macías Parra, Instituto Nacional de Pediatría, México; Gerardo Martínez Aguilar, IMSS, Durango, México; Abiel Mascareñas de los Santos, Centro Médico Hidalgo, Monterrey, Nuevo León, México; Sarbelio Moreno Espinosa, Hospital Infantil de México Federico Gómez, México; Aarón Pacheco Ríos, Hospital Infantil de México Federico Gómez, México; David Prado Cohrs, Universidad Francisco Marroquín, Guatemala; Miguel Angel Rodriguez Weber, Instituto Nacional de Pediatría, México; Luis Romano Mazzotti, Dirección Médica Vacunas, GlaxoSmithKline México; Erick Rosales Uribe, Hospital Infantil de México Federico Gómez, México; José Sifuentes Osornio, Instituto Nacional de Ciencias Médicas y Nutrición Salvador Zubirán, México; Rolando Ulloa-Gutiérrez, Hospital Nacional de Niños de Costa Rica "Dr. Carlos Sáenz Herrera”, San José, Costa Rica; Alberto Villaseñor Sierra, Centro de Investigación Biomédica de Occidente, IMSS, México
}

Fecha de recibido: 30 de agosto de 2010 • Fecha de aprobado: 2 de diciembre de 2010

Solicitud de sobretiros: Dr. Sarbelio Moreno Espinosa. Departamento de Infectología,

Hospital Infantil de México Federico Gómez. Dr. Márquez 162, col. Doctores. 06720 Delegación Cuauhtémoc, México DF.

Correo electrónico: sarbelio.moreno@gmail.com 
T a tos ferina es una enfermedad infecciosa causada $\perp$ por la Bordetella pertussis que es un bacilo aerobio, Gram-negativo, pleomórfico, no móvil, que afecta exclusivamente al ser humano y se transmite por partículas de secreciones respiratorias de personas infectadas. La $B$. pertussis se adhiere a las células ciliadas del epitelio nasofaríngeo y del árbol traqueo-bronquial mediante las moléculas de adhesión: hemaglutinina filamentosa (HFA), fimbrias, pertactina, y otras proteínas de superficie. Estas, junto con la toxina pertussis (TP), la citotoxina traqueal (CTT), la toxina dermonecrótica (TDN) y la toxina de adenil-ciclasa (TAC), constituyen algunos de los determinantes de patogenicidad. Las manifestaciones clínicas de la tos ferina varían según el huésped, y van desde la presencia de tos paroxística con estridor inspiratorio, periodos de apnea y tos emetizante, hasta síntomas leves que pueden ser confundidos con infecciones virales de las vías respiratorias. ${ }^{1,2}$

La infección por B. pertussis continúa siendo un problema de salud pública, incluso en países desarrollados con buenas coberturas de vacunación. ${ }^{3}$ La evidencia actual confirma que esta enfermedad continúa causando muertes en neonatos vulnerables y lactantes con esquemas de vacunación incompletos. ${ }^{4-6}$ Varios países han reportado un incremento en los casos de infección por B. pertussis durante la década pasada, asociada con cambios en la frecuencia reportada por grupos de edad. Se ha identificado un mayor porcentaje de esta enfermedad en adolescentes y adultos, ${ }^{7-12}$ que a su vez constituyen una fuente de infección mayor en los lactantes., ${ }^{4} 13-18$

Entre los factores que complican el conocimiento de la incidencia real, transmisión y carga de enfermedad de la infección por $B$. pertussis se encuentran:

1. Los adolescentes y adultos con un cuadro de tos persistente rara vez acuden a consulta. ${ }^{19}$

2. No se hace el diagnóstico o cuando se hace, no se reporta. ${ }^{20-22}$

3. Creencia de que esta enfermedad sólo afecta a niños, y aunque los síntomas sean típicos, no se diagnostica. ${ }^{23}$

4. Sistemas de vigilancia epidemiológica deficientes. ${ }^{24}$

5. Definiciones de caso inconsistentes. ${ }^{3,25,26}$

6. La enfermedad puede confundirse con otra infección respiratoria alta o baja. ${ }^{27}$

7. Técnicas de laboratorio diagnóstico no sensibles, no estandarizadas, mal realizadas o no disponibles. ${ }^{24}$

\section{Cambios en el patrón epidemiológico}

En la era prevacunación, la tos ferina era considerada una enfermedad endémica con picos epidémicos cada
2 a 5 años. ${ }^{28-30}$ En EUA se estimó que la incidencia de la enfermedad era en promedio de 157 casos por 100000 habitantes y ocurría casi exclusivamente en niños no vacunados. ${ }^{31,32}$ La introducción de la vacuna DPT de células enteras (décadas de 1940 y 1950) fue altamente efectiva, y para los años setenta la tasa de reporte de la infección disminuyó cerca de 150 veces y se mantuvo entre 0.5 y 1 caso por 100000 habitantes. ${ }^{33}$ Entre 1982 y 2002 se observó un incremento linear en la tasa de reporte de pertussis que llegó hasta 3.1 casos por 100000 habitantes. ${ }^{34,35}$ Esta tasa aumentó notablemente hasta 8.8 casos por 100000 habitantes en 2004. Estudios realizados en diferentes periodos (1962-1982 y 1983-2004) en EUA y Reino Unido (RU) señalan la persistencia del patrón endémico con picos epidémicos cada 2 a 5 años, y algunos autores sugieren que la enfermedad endémica en adolescentes y adultos es la responsable de éstos. $29,36,37$

Se estima que cada año se presentan entre $20 \mathrm{y}$ 50 millones de casos de $B$. pertussis en el mundo con aproximadamente 20000 a 40000 muertes al año, de las cuales $90 \%$ ocurre en países en desarrollo, la mayoría en lactantes de edad muy temprana para haber sido vacunados o tener un esquema completo de vacunación. ${ }^{33,38,39}$

La inmunidad después de la vacunación tiene una duración limitada. Con la vacuna DTP de células completas y vacunas DTPa pediátricas, la inmunidad empieza a disminuir después de 3 a 5 años, y no existe protección demostrable de los 10 a los 12 años. ${ }^{7}$ Con la vacuna DTP acelular (dTpa) no se ha determinado aún la duración de la protección, pero los valores de anticuerpos postvacunación sugieren que la protección dura entre 5 y 10 años. ${ }^{7}$ Reportes recientes sugieren que la inmunidad posterior a la infección natural por $B$. pertussis no es mayor que la que se obtiene por inmunización. ${ }^{28,40}$ En estudios llevados a cabo en Canadá y $\mathrm{RU}$, donde se evaluaron grupos de niños menores de 16 años con tos prolongada ( $\geq 14$ días), se encontró tos ferina en $35 \%$ de los adolescentes y que entre 60 y $80 \%$ habían sido inmunizados en la infancia. ${ }^{41,42}$

A continuación enumeramos las posibles causas del aumento en los reportes de tos ferina:

1. Cambios genéticos en la B. pertussis que hacen que las vacunas sean menos efectivas. ${ }^{43,44}$

2. Vacunas actuales menos eficaces que las anteriores. ${ }^{9}$

3. Disminución de la inmunidad inducida por las vacunas. ${ }^{45-48}$

4. Mayor preocupación por esta infección de parte de las autoridades en salud, los médicos y en algunos países desarrollados, la población en general. 
5. Mayor eficiencia de los laboratorios y mejores métodos para confirmación del diagnóstico en algunos países.

6. Mayor capacidad para reconocer la enfermedad en adolescentes y adultos. ${ }^{47,49}$

\section{Carga de enfermedad en México}

En México se han realizado al menos cuatro encuestas seroepidemiológicas de detección de anticuerpos antipertussis en población menor de 15 años; la última fue hace 22 años, por lo que los datos con que contamos para calcular la seroprevalencia de la enfermedad no corresponden al estado actual de la tos ferina en México. ${ }^{50-53} \mathrm{En}$ las tres seroencuestas realizadas entre los años sesenta y setenta se utilizaron diferentes métodos serológicos y un tamaño de muestra cada vez más grande. Se encontraron porcentajes de seropositividad cada vez mayores: en $1965,15.3 \% ; 1966,24.7 \%$ y en $1973,33 \%$. Los porcentajes de seropositividad variaron por edad y dosis recibidas de vacuna, pero a pesar de haberse utilizado diferentes técnicas, se aprecia un incremento en la seropositividad, probablemente debido al incremento en las coberturas de vacunación, pero también pudiera haber sido por una mejora en los métodos de detección.

En la última seroencuesta llevada a cabo en México en 1987, se determinaron aglutininas en 25666 muestras de sueros de niños de 1 a 15 años de edad en los diferentes estados de la República. Se consideraron positivos los títulos iguales o mayores a 1:16 y 1:32, y se encontró un 65 y $40 \%$ de seropositividad para cada dilución, respectivamente, con prevalencias mayores en las entidades del norte y centro de la República, así como en las mujeres, y títulos menores en los niños residentes en áreas rurales o los que fueron clasificados con nivel socioeconómico bajo. ${ }^{53}$

En un reporte de un brote de tos ferina en 1988 en una comunidad de Veracruz de 461 habitantes, se confirmaron $85(18.5 \%)$ casos por serología, donde el grupo más afectado es el de 7 a 11 meses, y como factores de riesgo se reconocieron la convivencia de 10 personas o más en la misma habitación y tener contacto con un enfermo. En ese mismo año se documentó una cobertura de vacunación de $6 \%$ en dicha comunidad. ${ }^{54}$

Entre 2002 y 2003 se realizó un estudio transversal para determinar la frecuencia de tos prolongada $(>14$ días) en 14 escuelas secundarias de la Ciudad de México, donde 12273 adolescentes fueron evaluados. Se documentaron 62 casos de tos prolongada (5 por 1000$)$. Entre esos sujetos se documentaron 20 (32.8\%) casos positivos para B. pertussis mediante la reacción en cadena de la polimerasa (PCR). También fueron positivos 16 (10\%) de 152 contactos escolares y $8(27.6 \%)$ de 29 contactos familiares..$^{55}$

De acuerdo con los informes de la Dirección General de Epidemiología (DGE) en el año 2009 se notificaron hasta la semana epidemiológica 46, 180 casos probables de tos ferina. En el reporte por casos confirmados en la República mexicana, los estados con mayor prevalencia fueron Sonora con 95 casos, Jalisco con 25 casos, Nuevo León con 20 y el Estado de México y Durango con 7 cada uno. En el grupo de edad de menores de un año se reportaron 103 casos (57\%) y en el grupo de 1 a 4 años, 17 casos $(9 \%)$.

Como podemos ver, existe muy poca información sobre la epidemiología de la enfermedad en adolescentes y adultos. Los datos disponibles nos hacen suponer que el país presenta una situación similar a la que existe en otros países de América Latina. ${ }^{27}$

\section{Diagnóstico}

Los métodos de diagnóstico más comunes para la detección de B. pertussis son el cultivo, detección directa del antígeno mediante anticuerpos fluorescentes, PCR y demostración serológica (ELISA y aglutinación) que miden incremento en la titulación de los valores, o valores individuales muy altos. Factores como la exposición anterior a la bacteria, edad, administración de antibióticos, inmunización, tiempo de toma de la muestra y sofisticación del laboratorio, pueden afectar la sensibilidad y la especificidad de las pruebas individuales.

Históricamente, el cultivo ha sido considerado como el método diagnóstico estándar de oro en los niños, sin embargo, la sensibilidad de las pruebas diagnósticas guarda relación con la fase de la enfermedad en la que el paciente se encuentra. ${ }^{56} \mathrm{El}$ médico debe conocer las limitaciones de las diferentes pruebas actualmente disponibles en relación con la fase de la enfermedad para emitir un diagnóstico confirmatorio de infección por B. pertussis (cuadro I).

A continuación mostramos las ventajas y desventajas de las diferentes pruebas diagnósticas para la detección de enfermedad por B. pertussis ${ }^{57}$ (cuadro II).

Otro criterio importante que debe tomarse en cuenta para elegir la prueba adecuada para hacer el diagnóstico de tos ferina es el periodo clínico en que se encuentra (catarral, paroxístico o convalecencia) o, en el adulto, en que no son tan evidentes dichos periodos, en la semana de evolución después del inicio de la tos ${ }^{58}$ (figura 1). 
Cuadro I

UTILIDAD DE LOS DISTINTOS MÉTODOS DIAGNÓSTICOS DE ACUERDO CON LA FASE DE LA ENFERMEDAD

\begin{tabular}{lccc}
\multicolumn{1}{r}{ Fase } & Catarral & Paroxística & Convalecencia \\
Cultivo & ++ & $-/+$ & - \\
\hline PCR & ++ & ++ & - \\
\hline Serología & $-/+$ & ++ & ++ \\
\hline
\end{tabular}

\section{Cuadro clínico en diferentes grupos de edad}

\section{Cuadro clínico en lactantes y niños}

Las manifestaciones clínicas en lactantes y niños no inmunizados se agrupan en tres periodos: catarral, paroxística y convaleciente. La presencia de fiebre es rara durante todo el curso de la enfermedad, a excepción de la fase catarral, en que se puede presentar en bajo grado.

\section{Periodo catarral}

Esta primera fase dura desde pocos días hasta una semana. Las manifestaciones clínicas en esta fase son indistinguibles a las de una infección leve del tracto respiratorio alto y son: rinorrea, lagrimeo y tos seca moderada. Durante la transición a la fase paroxística, la tos incrementa en frecuencia y gravedad y ocurre en paroxismos.

\section{Periodo paroxístico}

Esta segunda fase puede durar entre 2 y 6 semanas y se caracteriza por 5 a 10 episodios de tos forzada durante una sola fase espiratoria. Se puede oír un estridor (whoop) al final de la tos paroxística, como un intento del niño de inspirar a través de una glotis estrechada y espasmódica. Estos accesos pueden ocurrir varias veces al día (docenas) y pueden ser más graves durante la noche.

Periodo convaleciente

En esta tercera fase la tos disminuye en frecuencia y gravedad. Este periodo dura alrededor de 2 semanas, pero puede tomar meses para que el lactante se reestablezca por completo. Pueden ocurrir exacerbaciones durante infecciones respiratorias virales subsecuentes.

\section{Cuadro clínico en adolescentes y adultos}

Los adolescentes y los adultos con síntomas moderados o asintomáticos rara vez son diagnosticados con tos ferina. Los principales padecimientos con que se ha confundido el diagnóstico de tos ferina en los adolescentes son infecciones virales, asma, sinusitis, rinitis alérgica, neumonía, tuberculosis y epilepsia..$^{59}$ Diferentes estudios han demostrado que la tos ferina se presenta con mayor frecuencia en mujeres. Son más frecuentes las complicaciones conforme aumenta la edad. ${ }^{41} \mathrm{~A}$ pesar de que las manifestaciones clínicas en los adolescentes y adultos son atípicas y limitadas a tos moderada, ${ }^{37,60}$ muchas veces presentan sintomatología grave. ${ }^{61}$

Cuadro II

VENTAJAS Y DESVENTAJAS DE LAS DIFERENTES PRUEBAS DIAGNÓSTICAS PARA LA DETECCIÓN DE ENFERMEDAD POR B. PERTUSSIS ${ }^{56,58}$

\begin{tabular}{|c|c|c|}
\hline Prueba & Ventaja & Desventaja \\
\hline Cultivo & $\begin{array}{l}\text { Disponible al momento } \\
\text { Estandarizada } \\
\text { Alta especificidad }\end{array}$ & $\begin{array}{l}\text { Baja sensibilidad después de semana 2-3 } \\
\text { Recolección de la nasofaringe } \\
\text { Medio de crecimiento especial }\end{array}$ \\
\hline DFA & $\begin{array}{l}\text { Fácil de realizar } \\
\text { La bacteria no tiene que estar viable } \\
\text { Se puede realizar en estadios tardíos }\end{array}$ & $\begin{array}{l}\text { No recomendable por baja sensibilidad y especificidad variable } \\
\text { Reactividad cruzada con otras bacterias }\end{array}$ \\
\hline PCR & $\begin{array}{l}\text { Organismo no necesita estar vivo } \\
\text { Se puede usar más tardío en la enfermedad que el cultivo } \\
\text { Más rápido que el cultivo }\end{array}$ & $\begin{array}{l}\text { Contaminación potencial en laboratorio: } \\
\text { Falsos positivos } \\
\text { Disponible en pocos laboratorios } \\
\text { Métodos no estandarizados }\end{array}$ \\
\hline Serología & $\begin{array}{l}\text { Diagnóstico: } \\
\text { Incremento } 2 \text { a } 4 \text { veces entre fase aguda y convaleciente } \\
\text { Un solo valor convaleciente muy alto }\end{array}$ & $\begin{array}{l}\text { No estandarizadas entre laboratorios } \\
\text { Muestra convaleciente difícil de obtener } \\
\text { Resultados difíciles de interpretar }\end{array}$ \\
\hline
\end{tabular}




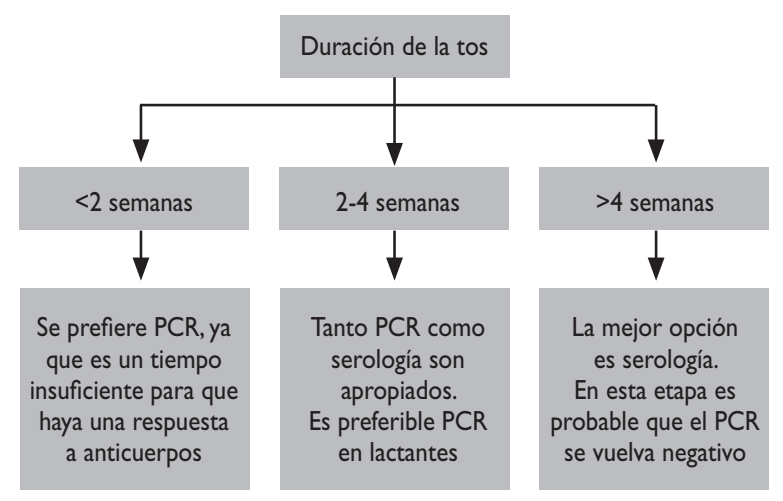

Figura I. Elección de prueba diagnóstica de acuerdo A LA DURACIÓN DE LA TOS ${ }^{56,58}$

\section{Recomendaciones de vacunación de refuerzo como vacunación masiva universal en adolescentes y adultos; recomendaciones actuales de vacunación}

\section{Experiencia de otros países}

Las vacunas contra tos ferina han sido parte de la inmunización rutinaria pediátrica por más de 50 años, lo que da por resultado una disminución importante de la incidencia de la enfermedad. ${ }^{62} \mathrm{Ni}$ la vacunación ni la infección natural inducen inmunidad a largo plazo, por lo que las reinfecciones son frecuentes. En la actualidad, en poblaciones con alta cobertura de vacunación, los adolescentes y adultos son a menudo fuente de infección para lactantes y niños no inmunizados o parcialmente inmunizados, quienes a su vez presentan el mayor riesgo de complicaciones y de mortalidad. ${ }^{63}$

En la actualidad, únicamente Austria, Australia, Canadá, Alemania y EUA recomiendan la vacuna contra pertussis en adolescentes como $5^{\mathrm{a}}$ o $6^{\mathrm{a}}$ dosis. ${ }^{64-74}$ Este refuerzo ha sido recomendado en Austria y EUA para la población general y en Alemania para grupos de alto riesgo como parientes, cuidadores de lactantes, y los trabajadores de la salud. ${ }^{64,71}$ En Latinoamérica, Argentina incorporó en 2009 una 6a dosis al esquema nacional de vacunación en el marco de los programas de salud escolar, a los 11 años de edad. En Costa Rica, en 2007 se introdujo la estrategia capullo de 1 dosis a las madres en el posparto inmediato. ${ }^{27}$

A pesar de que la mayoría de los países hoy día recomiendan una serie primaria de tres dosis en el primer año de vida, el número de dosis de refuerzo y el momento de administrarlas es altamente variable según el país, de acuerdo con cuatro factores fundamentales:

1. La realidad epidemiológica de cada país.

2. La duración de la protección conferida por la vacuna.

3. Los problemas logísticos de cada país.

4. Los costos adicionales que el nuevo esquema implica.

\section{Recomendaciones para recibir refuerzo de vacuna dTpa}

A pesar de que se desconoce el estado actual de inmunidad contra $B$. pertussis en México, ya que la última encuesta serológica se realizó en 1990, ${ }^{53}$ los cambios en el patrón epidemiológico de tos ferina son del conocimiento de las autoridades de salud del país, que publican en los Lineamientos Generales de Vacunación Universal y Semanas Nacionales de Salud de 2007 del Centro Nacional para la Salud de la Infancia y la Adolescencia (CeNSIA) la siguiente aseveración:

Sin embargo, según la experiencia de países con situación epidemiológica semejante a la de México, [se] ha[...] identificado un aumento de formas clínicas atípicas en adultos jóvenes que perdieron inmunidad y que constituyen fuentes de contagio potencialmente peligrosas. $^{75}$

En México no está contemplado un refuerzo de dTpa en el adolescente como parte del esquema de la Cartilla Nacional de Vacunación, pero existe la vacuna para su uso en la práctica privada desde hace varios años.

Existen diferentes recomendaciones para el uso de dTpa, que incluyen aplicación a adolescentes y adultos y otros grupos de alto riesgo, propuestos por el Comité Asesor de Prácticas en Vacunación (ACIP por sus siglas en inglés) de los CDC (Centers for Disease Control), la Academia Americana de Pediatría ${ }^{76}$ y otros organismos no oficiales como la Iniciativa Global de Pertussis (GPI) ${ }^{68,73,74}$ y el Consenso para el Refuerzo contra Pertussis en Europa (COPE). ${ }^{77}$ Las recomendaciones son muy similares entre las distintas entidades, con algunas variaciones debido a los diferentes grupos de población a que se refieren.

La Sociedad de Medicina del Adolescente de EUA sostiene el uso de la dTpa entre todos los adolescentes y adultos jóvenes en edades de 10 a 25 años. El único camino efectivo para disminuir el riesgo de transmisión de la enfermedad a neonatos y lactantes vulnerables es prevenir la enfermedad entre adolescentes y adultos 
jóvenes, quienes están en mayor riesgo de contraer la enfermedad. ${ }^{33}$

La disponibilidad de una dosis de refuerzo para los trabajadores de la salud puede ser el modo más efectivo de reducir el riesgo de infección y transmisión en instituciones de salud. Se sabe que la vacunación en este grupo es costo-efectiva, pero se requieren futuros estudios sobre la duración de la inmunidad y el impacto que dicha vacunación tiene sobre el manejo de la exposición a pertussis. ${ }^{78}$

El grupo de mayor riesgo de hospitalización, complicaciones y muerte lo constituyen los lactantes menores de 3 meses de edad, pese a las buenas tasas de cobertura en niños. Por esta razón se han establecido estrategias directas e indirectas para proteger a este grupo vulnerable.

A pesar de que la transmisión transplacentaria de IgG específica para pertussis es de $50 \%$, los niveles de IgG al nacimiento declinan rápidamente en los dos primeros meses de vida, por lo que se ha calculado que la vida media de los anticuerpos contra PT y FHA es de 36 a 40 días, respectivamente. Hasta la fecha, la experiencia acumulada en varios países con el uso de vacuna dTpa no ha demostrado efectos adversos serios maternos o neonatales. Estos datos soportan una base racional para la evaluación futura de inmunización neonatal a través de la madre como un posible abordaje para prevenir pertussis en infantes menores de tres meses. ${ }^{79}$

Al tomar en cuenta las diferentes recomendaciones de vacunación contra $B$. pertussis en el lactante, adolescente, adulto, personal de salud y grupos de alto riesgo, y la evidencia existente para posibles grupos poblacionales en que se puede incidir para evitar la diseminación de dicha enfermedad, se ha sugerido el siguiente cuadro en el que se muestran los diferentes niveles en que se puede incidir para frenar la transmisión de B. pertussis ${ }^{33}$ (cuadro III).

\section{Implementación en México de la vacunación en adolescentes y adultos}

Ha quedado bien establecido que, en países con altas coberturas vacunales como México, la tos ferina es hoy en día una enfermedad de dos grupos poblacionales: los mayores de 10 años de edad y los niños pequeños no vacunados o parcialmente vacunados (infectados por el contacto con niños mayores, adolescentes y adultos). ${ }^{4-6,30,89}$ En los primeros, la morbilidad es significativa con casos graves ocasionales. En los segundos, las posibilidades de cuadros serios, hospitalización y muerte son altas. Así que la necesidad de vacunar a adolescentes y adultos ha quedado claramente definida. ¿Cómo llevarla a cabo en países como México?

Los participantes en este consenso consideramos que la implementación descansa en tres pilares fundamentales, que se encuentran estrechamente relacionados:

1. Educación

2. Estudios epidemiológicos

3. Estrategias definidas de introducción de la vacuna

Mencionamos en la introducción que, con frecuencia, los médicos no hacemos el diagnóstico de la enfermedad, ${ }^{20-22}$ sobre todo en adolescentes y adultos donde el cuadro clínico es atípico. ${ }^{37}$ Ante la creencia de que la enfermedad sólo afecta a niños, ${ }^{23}$ se confunde el diagnóstico con múltiples entidades. ${ }^{59}$ En otras palabras, si no existe conciencia del problema en el gremio médico, tampoco se visualizará la necesidad de la vacunación. Si los médicos no tenemos esta conciencia, tampoco la tendrán algunas autoridades de salud ni el público en general. Así que la educación del gremio médico y el personal de salud constituye el terreno fértil donde la iniciativa de vacunación puede florecer. Este proceso de educación debe tener una participación multisectorial,

Cuadro III

Objetivos de las distintas estrategias de VACunación CONTRA B. PERTUSSIS

\begin{tabular}{|c|c|c|}
\hline \multirow{2}{*}{ Inmunización } & \multicolumn{2}{|c|}{ Objetivo } \\
\hline & Primario & Secundario \\
\hline Universal ${ }^{80}$ adolescentes & $\begin{array}{l}\text { Reducir incidencia en adolescente } \\
\text { Promover inmunidad de rebaño }\end{array}$ & Reducir transmisión a lactantes \\
\hline Universal $^{69,81}$ adultos & $\begin{array}{l}\text { Reducir incidencia en adulto } \\
\text { Promover inmunidad de rebaño }\end{array}$ & $\begin{array}{l}\text { Reducir transmisión a otros } \\
\text { (recién nacidos y lactantes) }\end{array}$ \\
\hline Selectiva alrededor recién nacido 82,83 & Reducir transmisión en lactantes & Reducir morbilidad en la familia \\
\hline Selectiva ${ }^{84,85}$ trabajadores de salud & Reducir transmisión en pacientes & Reducir morbilidad en trabajadores de salud \\
\hline Selectiva ${ }^{86,87}$ trabajadores de salud & Reducir transmisión en niños & Reducir morbilidad en trabajadores de salud \\
\hline Recién nacidos al nacimiento & Reducir transmisión en lactantes & Reducir morbilidad en la niñez \\
\hline Madre durante el embarazo ${ }^{88}$ & Reducir transmisión en recién nacidos & Reducir morbilidad en las madres \\
\hline
\end{tabular}


que involucre autoridades de salud, asociaciones médicas e industria farmacéutica.

Los estudios epidemiológicos son también fundamentales para generar una necesidad de prevención. Aunque a veces pareciera que se quiere demostrar lo demostrado, es difícil hacer recomendaciones sólidas y convencer incrédulos en un país donde la última encuesta seroepidemiológica se realizó hace 22 años. ${ }^{50-53}$ Adicionalmente decíamos que los tres pilares se encuentran estrechamente relacionados. Los datos epidemiológicos poseen intrínsecamente un valor educacional y constituyen un aporte invaluable en el proceso de generación de conciencia del problema de pertussis.

Llegamos finalmente a las estrategias de introducción. La experiencia en algunos países latinoamericanos ha mostrado que la introducción escalonada tiene una tasa de éxito alta, comparada con la ambiciosa introducción masiva, donde por desear tener muchos alcances no se obtiene ninguno. Por ello, este grupo de consenso piensa que la implementación debiera tener el siguiente proceso secuencial:

1. Fomento del uso rutinario de las vacunas acelulares para adolescentes y adultos en las diferentes prácticas privadas.

2. Aplicación de la vacuna por parte de las autoridades de salud en un grupo definido de riesgo -ya sea en adolescentes, individuos en contacto con menores de 12 meses y mujeres antes del embarazo o en el posparto inmediato (estrategia capullo)-.

3. Vacunación universal que incluya adolescentes, adultos, individuos en contacto con menores de 12 meses, personal de salud y mujeres antes del embarazo o en el posparto inmediato.

Debemos hacer notar que algunas de las estrategias no son excluyentes. Por ejemplo, si se inicia con la "estrategia capullo", ésta no debiera ser interrumpida al iniciar la vacunación de adolescentes, sino que debe continuar hasta que la cobertura vacunal en adultos sea suficiente para proporcionar inmunidad de rebaño.

Este grupo de consenso espera que la presente publicación haga una contribución real que conduzca a la disminución de la carga de tos ferina y sus consecuencias, en México y Latinoamérica.

Declaración de conflicto de intereses: El Dr. James Cherry declara haber recibido honorarios por consultoría sobre vacunas de tos ferina de GSK y Sanofi-Pasteur. La Dra. Yolanda Cervantes declara ser empleada de GSK México. El Dr. Luis Romano declara ser empleado de GSK México. El Dr. David Prado declara haber recibido honorarios como investigador y haber participado en reuniones de expertos para GSK, Novartis y Sanofi-Pasteur. El Dr.Ulloa-Gutiérrez declara haber sido conferencista y haber participado en reuniones de expertos para GSK, Sanofi-Pasteur y Wyeth. Los demás autores declararon no tener conflicto de intereses.

\section{Referencias}

I. Mattoo S, Cherry J. Molecular pathogenesis, epidemiology, and clinical manifestations of respiratory infections due to Bordetella pertussis and other Bordetella subspecies. Clin Microbiol Rev 2005; 18:326.

2. Versteegh FGA, Schellekens JFP, Fleer A, Roord J. Pertussis: a concise historical review including diagnosis, incidence, clinical manifestations and the role of treatment and vaccination in management. Rev Med Microbiol 2005;16:79-89.

3. World Health Organization. Dept. of Vaccines and Other Biologicals. Pertussis surveillance: a global meeting : Geneva, 16-18 October 2000. Geneva:World Health Organization, Dept. of Vaccines and Biologicals, 2001. 4. Tanaka M,Vitek CR, Pascual FB, Bisgard KM, Tate JE, Murphy TV.Trends in pertussis among infants in the United States, 1980-1999. JAMA 2003;290:2968-2975.

5. Murphy TV, Slade BA, Broder KR, Kretsinger K, Tiwari T, Joyce PM, et al. Prevention of pertussis, tetanus, and diphtheria among pregnant and postpartum women and their infants recommendations of the Advisory Committee on Immunization Practices (ACIP). MMWR Recomm Rep 2008;57:I-5I.

6. Celentano LP, Massari M, Paramatti D, Salmaso S, Tozzi AE. Resurgence of pertussis in Europe. Pediatr Infect Dis J 2005;24:761-765.

7. Halperin SA. The control of pertussis--2007 and beyond. N Engl J Med 2007;356:1 I0-1 I3.

8. Aguas R, Goncalves G, Gomes MG. Pertussis: increasing disease as a consequence of reducing transmission. Lancet Infect Dis 2006;6: I I2- I I7. 9. Cherry JD. The epidemiology of pertussis: a comparison of the epidemiology of the disease pertussis with the epidemiology of Bordetella pertussis infection. Pediatrics 2005; I 15: I 422-1 427.

10. Brooks DA, Clover R. Pertussis infection in the United States: role for vaccination of adolescents and adults. J Am Board Fam Med 2006; 19:603-6II. II. Wilder-Smith A, Ng S, Earnest A. Seroepidemiology of pertussis in the adult population of Singapore. Ann Acad Med Singapore 2006;35:780-782. 12. Hewlett EL, Edwards KM. Clinical practice. Pertussis--not just for kids. N Engl J Med 2005;352:1215-1222.

13. Cortese MM, Baughman AL, Zhang R, Srivastava PU, Wallace GS. Pertussis hospitalizations among infants in the United States, 1993 to 2004. Pediatrics 2008;121:484-492.

14. Healy CM, Rench MA, Edwards KM, Baker CJ. Pertussis serostatus among neonates born to Hispanic women. Clin Infect Dis 2006;42: 1439-1442. 15. Bisgard KM, Pascual FB, Ehresmann KR, Miller CA, Cianfrini C, Jennings $\mathrm{CE}$, et al. Infant pertussis: who was the source? Pediatr Infect Dis J 2004:23:985-989.

16. Moraga F, Roca J, Mendez C, Rodrigo C, Pineda V, Martinez A, et al. Epidemiology and surveillance of pertussis among infants in Catalonia, Spain, during 1997-200I. Pediatr Infect Dis J 2005;24:5 I0-5 I3.

17. Wendelboe AM, Njamkepo E, Bourillon A, Floret DD, Gaudelus ], Gerber M, et al. Transmission of Bordetella pertussis to young infants. Pediatr Infect Dis J 2007;26:293-299.

18. Baptista PN, Magalhã $f E s$ VS, Rodrigues LC. The role of adults in household outbreaks of pertussis. Int J Inf Dis 2009: I-4.

19. Jenkinson D. Natural course of 500 consecutive cases of whooping cough: a general practice population study. BMJ 1995;3 10:299-302. 20. Deville JG, Cherry JD, Christenson PD, Pineda E, Leach CT, Kuhls TL, et al. Frequency of unrecognized Bordetella pertussis infections in adults. Clin Infect Dis 1995;21:639-642.

2I. Gilberg S, Njamkepo E, Du Chatelet IP, Partouche H, Gueirard P, Ghasarossian C, et al. Evidence of Bordetella pertussis infection in adults presenting with persistent cough in a french area with very high wholecell vaccine coverage.J Infect Dis 2002; 186:415-4I8.

22. Von Konig CH, Halperin S, Riffelmann M, Guiso N. Pertussis of adults and infants. Lancet Infect Dis 2002;2:744-750. 
23. Herwaldt LA. Pertussis in adults. What physicians need to know. Arch Intern Med 1991;|51:1510-1512.

24. Cherry JD, Grimprel E, Guiso N, Heininger U, Mertsola J. Defining pertussis epidemiology: clinical, microbiologic and serologic perspectives. Pediatr Infect Dis J 2005;24:S25-34.

25. Patriarca PA, Biellik RJ, Sanden G, Burstyn DG, Mitchell PD, Silverman $P R$, et al. Sensitivity and specificity of clinical case definitions for pertussis. Am J Public Health 1988;78:833-836.

26. Pertussis outbreak --Vermont, 1996. MMWR Morb Mortal Wkly Rep 1997; 46:822-826.

27. Ulloa-Gutierrez R,Avila-Aguero M. Pertussis in Latin America: current situation and future vaccination challenges. Expert Rev Vaccines 2008;7:1569-1580.

28. Cherry JD. Epidemiological, clinical, and laboratory aspects of pertussis in adults. Clin Infect Dis 1999;28 Suppl 2:SI I2-II7.

29. Cherry JD.The epidemiology of pertussis and pertussis immunization in the United Kingdom and the United States: a comparative study. Curr Probl Pediatr 1984;14:1-78.

30. Mink CM, Cherry JD, Christenson P, Lewis K, Pineda E, Shlian D, et al. A search for Bordetella pertussis infection in university students. Clin Infect Dis 1992;|4:464-47|.

31. Cherry JD. Pertussis in the preantibiotic and prevaccine era, with emphasis on adult pertussis. Clin Infect Dis 1999;28 Suppl 2:S107-I I I. 32. Pertussis--United States, 1997-2000. MMWR Morb Mortal Wkly Rep 2002;51:73-76.

33. Forsyth K. Pertussis, Still a Formidable Foe. Clin Infect Dis 2007;45. 34. Cherry J.The science and fiction of the» resurgence» of pertussis. Pediatrics 2003; I 2:405.

35. Groseclose SL, Brathwaite WS, Hall PA, Connor FJ, Sharp P,Anderson WJ, et al. Summary of notifiable diseases--United States, 2002. MMWR Morb Mortal Wkly Rep 2004;5I:I-84.

36. Miller E, Fleming DM,Ashworth LA, Mabbett DA, Vurdien JE, Elliott TS. Serological evidence of pertussis in patients presenting with cough in general practice in Birmingham. Commun Dis Public Health 2000;3:132-134. 37. Aoyama T, Takeuchi Y, Goto A, Iwai H, Murase Y, Iwata T. Pertussis in adults. Am J Dis Child 1992;146:163-166.

38. Crowcroft NS, Stein C, Duclos P, Birmingham M. How best to estimate the global burden of pertussis? Lancet Infect Dis 2003;3:4I3-4I8.

39. Tan T,Trindade E, Skowronski D. Epidemiology of pertussis. Pediatr Infect Dis J 2005;24:SI0-18.

40. Cherry JD, Beer T, Chartrand SA, DeVille J, Beer E, Olsen MA, et al. Comparison of values of antibody to Bordetella pertussis antigens in young German and American men. Clin Infect Dis 1995;20: I 27I-I 274. 4I. Senzilet LD, Halperin SA, Spika JS, Alagaratnam M, Morris A, Smith B. Pertussis is a frequent cause of prolonged cough illness in adults and adolescents. Clin Infect Dis 200I;32:169I-1697.

42. Harnden A, Grant C, Harrison T, Perera R, Brueggemann AB, MayonWhite $R$, et al. Whooping cough in school age children with persistent cough: prospective cohort study in primary care. BMJ 2006;333:174-177. 43. Mooi FR, van Loo IH, King AJ.Adaptation of Bordetella pertussis to vaccination: a cause for its reemergence? Emerg Infect Dis 200I;7:526-528. 44. King AJ, van Gorkom T, van der Heide HG, Advani A, van der Lee S. Changes in the genomic content of circulating Bordetella pertussis strains isolated from the Netherlands, Sweden, Japan and Australia: adaptive evolution or drift? BMC Genomics 2010; I :64.

45. Taranger J, Trollfors B, Bergfors E, Knutsson N, Lagergard T, Schneerson $R$, et al. Immunologic and epidemiologic experience of vaccination with a monocomponent pertussis toxoid vaccine. Pediatrics 200I; I08:EI I5. 46. Black S. Epidemiology of pertussis. Pediatr Infect Dis J 1997; 16:S85-89. 47. Pebody RG, Gay NJ, Giammanco A, Baron S, Schellekens J,Tischer A, et al. The seroepidemiology of Bordetella pertussis infection in Western Europe. Epidemiol Infect 2005; 133:159-17|

48. Pichichero ME. Booster vaccinations: can immunologic memory outpace disease pathogenesis? Pediatrics 2009;124:1633-1641.
49. Yih WK, Lett SM, des Vignes FN, Garrison KM, Sipe PL, Marchant CD. The increasing incidence of pertussis in Massachusetts adolescents and adults, 1989-1998.J Infect Dis 2000;|82:|409-1416.

50. Gutierrez G, Kumate J, Mendia AM. Immunological survey of the infantile population of Mexico, D.F. II. Investigation of antibodies against Hemophilus pertussis. Bol Med Hosp Infant Mex 1965;22:833-836. 51. Perez Miravete A, Perez de la Mora S. Serological tests to investigate parapertussis infections in Mexico. Rev Invest Salud Publica 1966;26:235-244. 52. Gutierrez G, Ruiz-Gomez J. Seroepidemiology of 10 infectious diseases in children in Mexico City. Measles, rubella, parotitis, typhoid, whooping cough, amebiasis, influenza and adenovirus infections, Mycoplasma pneumoniae and herpes simplex. Gac Med Mex 1973;105:529-540.

53. Sapian-Lopez LA, Valdespino JL, Salvatierra B, Tapia-Conyer R, Gutierrez G, Macedo J, et al. Seroepidemiology of whooping cough in Mexico. Salud Publica Mex 1992;34:177-185.

54. Navarrete-Navarro S, Gonzalez-Romero M, Ruiz-Matus C, CasasolaFlores J. Epidemiologic study of a whooping cough outbreak in Rincon Grande, Veracruz. Bol Med Hosp Infant Mex 1990;47:304-3I2.

55. Tomé-Sandoval P,Torres-Arreola LP, Romero-Quechol G, GuiscafréGallardo H. Bordetella pertussis in adolescents students in Mexico City. Rev Saúde Pública 2008;42:679-683.

56. Tozzi AE, Celentano LP, Ciofi degli Atti ML, Salmaso S. Diagnosis and management of pertussis. CMAJ 2005; 172:509-515.

57. Greenberg DP. Pertussis in adolescents: increasing incidence brings attention to the need for booster immunization of adolescents. Pediatr Infect Dis J 2005;24:721-728.

58. Sintchenko V.The re-emergence of pertussis: implications for diagnosis and surveillance. N SW Public Health Bull 2008;19:143-| 45.

59. Eidlitz-Markus T, Mimouni M, Zeharia A. Pertussis symptoms in adolescents and children versus infants: the influence of vaccination and age. Clin Pediatr 2007;46:718-723.

60. Yaari E, Yafe-Zimerman Y, Schwartz SB, Slater PE, Shvartzman P,Andoren $\mathrm{N}$, et al. Clinical manifestations of Bordetella pertussis infection in immunized children and young adults. Chest 1999; I 15:1254-1258. 61. Robertson PW, Goldberg H, Jarvie BH, Smith DD, Whybin LR. Bordetella pertussis infection: a cause of persistent cough in adults. Med J Aust 1987; 146:522-525.

62. Maciosek MV, Coffield AB, Edwards NM, Flottemesch TJ, Goodman MJ, Solberg LI. Priorities among effective clinical preventive services: results of a systematic review and analysis. Am J Prev Med 2006;31:52-61.

63. Hitchcock WP. Rationale for use of Tdap booster vaccines for adolescent immunization: overview of efficacy, safety, and clinical use. Clin Pediatr 2006;45:785-794.

64. Rendi-Wagner P, Paulke-Korinek M, Stanek G, Khanakah G, Kollaritsch $\mathrm{H}$. Impact of a pertussis booster vaccination program in adolescents and adults on the epidemiology of pertussis in Austria. Pediatr Infect Dis J 2007;26:806-810

65. Ward Jl, Cherry JD, Chang S-J, Partridge S, Lee H,Treanor J, et al. Efficacy of an acellular pertussis vaccine among adolescents and adults. $\mathrm{N}$ Engl J Med 2005;353: I555-1563.

66. Zepp F, Knuf M, Habermehl P, Mannhardt-Laakmann W, Howe B, Friedland LR. Safety of reduced-antigen-content tetanus-diphtheriaacellular pertussis vaccine in adolescents as a sixth consecutive dose of acellular pertussis-containing vaccine.J Pediatr 2006; I49:603-610. 67. Halperin SA. Canadian experience with implementation of an acellular pertussis vaccine booster-dose program in adolescents: implications for the United States. Pediatr Infect Dis J 2005;24:S I I - I 46.

68. Wirsing von Konig CH, Campins-Marti M, Finn A, Guiso N, Mertsola J, Liese J. Pertussis immunization in the global pertussis initiative European region: recommended strategies and implementation considerations. Pediatr Infect Dis J 2005;24:S87-92.

69. Kretsinger K, Broder KR, Cortese MM, Joyce MP, Ortega-Sanchez I, Lee GM, et al. Preventing tetanus, diphtheria, and pertussis among adults: 
use of tetanus toxoid, reduced diphtheria toxoid and acellular pertussis vaccine recommendations of the Advisory Committee on Immunization Practices (ACIP) and recommendation of ACIP, supported by the Healthcare Infection Control Practices Advisory Committee (HICPAC), for use of Tdap among health-care personnel. MMWR Recomm Rep 2006;55: I-37.

70. Pichichero ME, Blatter MM, Kennedy WA, Hedrick J, Descamps D, Friedland LR.Acellular pertussis vaccine booster combined with diphtheria and tetanus toxoids for adolescents. Pediatrics 2006; I I7:I084-1093. 7I. Zepp F, Habermehl P, Knuf M, Mannhardt-Laakman W, Howe B, Friedland LR. Immunogenicity of reduced antigen content tetanusdiphtheria-acellular pertussis vaccine in adolescents as a sixth consecutive dose of acellular pertussis-containing vaccine.Vaccine 2007;25:5248-5252. 72. Pichichero ME, Rennels MB, Edwards KM, Blatter MM, Marshall GS, Bologa $\mathrm{M}$, et al. Combined tetanus, diphtheria, and 5-component pertussis vaccine for use in adolescents and adults. JAMA 2005;293:3003-30I I. 73. Tan T, Halperin S, Cherry JD, Edwards K, Englund JA, Glezen P, et al. Pertussis immunization in the global pertussis initiative North American region: recommended strategies and implementation considerations. Pediatr Infect Dis J 2005;24:S83-86.

74. Forsyth K, Nagai M, Lepetic A, Trindade E. Pertussis immunization in the global pertussis initiative international region: recommended strategies and implementation considerations. Pediatr Infect Dis J 2005;24:S93-97.

75. Centro Nacional para la Salud de la Infancia y la Adolescencia (CeNSIA). Lineamientos Generales Vacunación Universal y Semanas Nacionales de Salud 2007. [Accesado 2010 octubre 20]. Disponible en : www. enfermedadesinfecciosas.com/files/vacunacion_universal_lineamientos.pdf. 76. America Academy of Pediatrics Commitee on Infectious D. Prevention of pertussis among adolescents: recommendations for use of tetanus toxoid, reduced diphtheria toxoid, and acellular pertussis (Tdap) vaccine. Pediatrics 2006; I 17:965-978.

77. Zepp F, Bernatowska E, Guiso N, Heininger U, Mertsola J, Roord J, et al. Consensus on pertussis booster vaccination in Europe. In: ESPID. Brussels, Belgium; 2009.
78. Sandora T], Gidengil CA, Lee GM. Pertussis vaccination for health care workers. Clin Microbiol Rev 2008;21:426-434.

79. Mooi F, Degreeff S. The case for maternal vaccination against pertussis. Lancet Infect Dis 2007;7:6 14-624.

80. Immunization of adolescents: recommendations of the Advisory Committee on Immunization Practices, the American Academy of Pediatrics, the American Academy of Family Physicians, and the American Medical Association. American Academy of Pediatrics Committee on Infectious Diseases. Pediatrics 1997;99:479-488.

8I. Cortese MM, Baughman AL, Brown K, Srivastava P.A "new age" in pertussis prevention new opportunities through adult vaccination. Am J Prev Med 2007;32: I77-I85.

82. Forsyth KD,Wirsing von Konig CH, Tan T, Caro J, Plotkin S. Prevention of pertussis: recommendations derived from the second Global Pertussis Initiative roundtable meeting.Vaccine 2007;25:2634-2642.

83. Van Rie A, Hethcote HW.Adolescent and adult pertussis vaccination: computer simulations of five new strategies. Vaccine 2004;22:3 I54-3 I65. 84. Peadon E, Cooper C.Whooping cough: are health-care workers putting children at risk? J Paediatr Child Health 2007;43:398-402. 85. Calugar A, Ortega-Sanchez IR, Tiwari T, Oakes L, Jahre JA, Murphy TV. Nosocomial pertussis: costs of an outbreak and benefits of vaccinating health care workers. Clin Infect Dis 2006;42:98I-988.

86. Forsyth K, Tan T, von Konig CH, Caro JJ, Plotkin S. Potential strategies to reduce the burden of pertussis. Pediatr Infect Dis J 2005;24:S69-74.

87. Schellekens J, von Konig CH, Gardner P. Pertussis sources of infection and routes of transmission in the vaccination era. Pediatr Infect Dis J 2005;24:S19-24

88. Lichty JA, Slavin B, Bradford WL. An Attempt to Increase Resistance to Pertussis in Newborn Infants by Immunizing Their Mothers during Pregnancy. J Clin Invest 1938; 17:613-621.

89. Strebel P, Nordin J, Edwards K, Hunt J, Besser J, Burns S, et al. Population-based incidence of pertussis among adolescents and adults, Minnesota, 1995-1996. J Infect Dis 200I;183:1353-1359. 